DRAFT VERSION SEPTEMBER 12, 2018

Preprint typeset using LTEX style emulateapj v. 5/2/11

\title{
SPONTANEOUS FORMATION OF SURFACE MAGNETIC STRUCTURE FROM LARGE-SCALE DYNAMO IN STRONGLY-STRATIFIED CONVECTION
}

\author{
YOUHEI MASADA ${ }^{1}$, AND TAKAYOSHI SANO $^{2}$ \\ Draft version September 12, 2018
}

\begin{abstract}
We report the first successful simulation of spontaneous formation of surface magnetic structures from a large-scale dynamo by strongly-stratified thermal convection in Cartesian geometry. The large-scale dynamo observed in our strongly-stratified model has physical properties similar to those in earlier weakly-stratified convective dynamo simulations, indicating that the $\alpha^{2}$-type mechanism is responsible for it. Additionally to the large-scale dynamo, we find that large-scale structures of the vertical magnetic field are spontaneously formed in the convection zone surface only for the case of strongly-stratified atmosphere. The organization of the vertical magnetic field proceeds in the upper convection zone within tens of convective turn-over time and band-like bipolar structures are recurrently-appeared in the dynamo-saturated stage. We examine possibilities of several candidates as the origin of the surface magnetic structure formation, and then suggest the existence of an as-yet-unknown mechanism for the self-organization of the large-scale magnetic structure, which should be inherent in the strongly-stratified convective atmosphere.
\end{abstract}

Subject headings: convection - magnetohydrodynamics (MHD) - Sun: magnetism -sunspots

\section{INTRODUCTION}

A long-standing goal of the solar interior physics is to reproduce active regions, composed mainly of the sunspots, self-consistently from magnetic fluxes generated in the solar interior. We now approach the subject from two different theoretical perspectives: one focuses on emergence and organization processes of the magnetic flux in the uppermost part of the convection zone $(\mathrm{CZ})$, and the other explores the flux generation and maintenance processes, i.e., the dynamo process, operating deeper down.

Several leading-edge numerical studies, which focus on the uppermost part of the solar $\mathrm{CZ}$, have succeeded to simulate spontaneous formations of concentrated magnetic structures reminiscent of active regions (e.g., Cheung et al. 2010; Stein \& Nordlund 2012; Rempel \& Cheung 2014; Käpylä et al. 2015). In these studies, the solar surface convection and its nonlinear interaction with the magnetic field were simulated in a more or less realistic manner with the steep density gradient just below the photosphere and/or the radiative transfer with the ionization in Cartesian domains. However, since some sort of the large-scale seed magnetic field has been assumed inconsistently as initial or boundary condition, the dynamo mechanism and its connection to the formation process of the active region were beyond the scope of these studies.

A growing body of evidence is accumulating to demonstrate that solar-like cyclic large-scale magnetic field is organized in global spherical-shell convections (e.g., Ghizaru et al. 2010; Käpylä et al. 2012; Masada et al. 2013; Yadav et al. 2015; Augustson et al. 2015). Despite some differences in the numerical setup and method, there is a common outcome of the convective dynamo in these studies: diffuse magnetic flux extending over the $\mathrm{CZ}$ and/or the tachocline instead of magnetic flux tubes expected in the standard solar dynamo paradigm (e.g., Charbonneau 2010, and references therein). Although the flux emergence like event from distributed mag-

\footnotetext{
${ }^{1}$ Department of Physics and Astronomy, Aichi University of Education; Kariya, Aichi 446-8501, Japan: E-mail: ymasada@auecc.aichi-edu.ac.jp

${ }^{2}$ Institute of Laser Engineering, Osaka University; Osaka 565-0871, Japan: E-mail: sano@ile.osaka-u.ac.jp
}

netic flux has been occasionally observed in some models (Nelson et al. 2013; Fan \& Fang 2014), its universality or feasibility in the Sun is still a matter of considerable debate.

There is still a large gap between the dynamo in the interior and the active region formation at the surface. Our study in this Letter would be a first step aiming to bridge the gap between them. By advancing our previous works of weaklystratified MHD convection (Masada \& Sano 2014a|b, hereafter MS14a,b), we perform convective dynamo simulation in a strongly-stratified atmosphere resembling the solar interior in Cartesian geometry. The spontaneous formation of largescale magnetic structures in the CZ surface self-consistently from the large-scale convective dynamo is reported.

\section{NUMERICAL SETUP}

Convective dynamo system is solved numerically in Cartesian domain. Our model covers only the $\mathrm{CZ}$ of depth $d_{\mathrm{cz}}$ $\left(0 \leq z \leq d_{\mathrm{cz}}\right)$ with omitting a stably-stratified layer below it, where $x$ - and $y$-axes are taken to be horizontal and $z$-axis is pointing downward. We set the width of the domain to be $W=4 d_{\mathrm{cz}}$.

We solve the fully-compressible MHD equations in the rotating frame of reference with a constant angular velocity of $\boldsymbol{\Omega}=-\Omega_{0} e_{z}$,

$$
\begin{aligned}
\frac{\partial \rho}{\partial t} & =-\nabla \cdot(\rho u), \\
\frac{\mathcal{D} u}{\mathcal{D} t} & =-\frac{\nabla P}{\rho}+\frac{J \times B}{\rho}-2 \boldsymbol{\Omega} \times u+\frac{\nabla \cdot \boldsymbol{\Pi}}{\rho}+g, \\
\frac{\mathcal{D} \epsilon}{\mathcal{D} t} & =-\frac{P \nabla \cdot u}{\rho}+\mathcal{Q}_{\text {heat }}, \\
\frac{\partial B}{\partial t} & =\nabla \times\left(u \times B-\eta_{0} J\right),
\end{aligned}
$$

with the viscous stress $\Pi$ and the heating term $\mathcal{Q}_{\text {heat }}$ of

$$
\Pi_{i j}=2 \rho \nu_{0} S_{i j}=\rho \nu_{0}\left(\frac{\partial u_{i}}{\partial x_{j}}+\frac{\partial u_{j}}{\partial x_{i}}-\frac{2}{3} \delta_{i j} \frac{\partial u_{i}}{\partial x_{i}}\right),
$$




$$
\mathcal{Q}_{\text {heat }}=\frac{\gamma \nabla \cdot\left(\kappa_{0} \nabla \epsilon\right)}{\rho}+2 \nu_{0} S^{2}+\frac{\mu_{0} \eta_{0} J^{2}}{\rho},
$$

where $\mathcal{D} / \mathcal{D} t$ is the total derivative, $\epsilon=c_{\mathrm{V}} T$ is the specific internal energy, $J=\nabla \times B / \mu_{0}$ is the current density with the vacuum permeability $\mu_{0}$, and $g=g_{0} e_{z}$ is the gravity of the constant $g_{0}$. The viscosity, magnetic diffusivity, and thermal conductivity are represented by $\nu_{0}, \eta_{0}$, and $\kappa_{0}$, respectively. A perfect gas law $P=(\gamma-1) \rho \epsilon$ with $\gamma=5 / 3$ is assumed. by

The initial hydrostatic state is polytropic stratification given

$$
\epsilon=\epsilon_{0}+\frac{g_{0} z}{(\gamma-1)(m+1)}, \text { and } \rho=\rho_{0}\left(\epsilon / \epsilon_{0}\right)^{m},
$$

with the initial surface internal energy $\epsilon_{0}$, surface density $\rho_{0}$, and the polytropic index $m=1.49$, providing the superadiabaticity of $\delta \equiv \nabla-\nabla_{\mathrm{ad}}=1.6 \times 10^{-3}$, where $\nabla_{\mathrm{ad}}=1-1 / \gamma$ and $\nabla=(\partial \ln T / \partial \ln P)$.

Normalization quantities are defined by setting $d_{\mathrm{cz}} / 2=g_{0}=$ $\rho_{0}=\mu_{0}=c_{p}=1$. The normalized pressure scale-height at the surface, defined by $\xi=H_{p} / d_{\mathrm{cz}}=(\gamma-1) \epsilon_{0} /\left(g_{0} d_{\mathrm{cz}}\right)$, controls the stratification level and is chosen here as $\xi=0.01$, yielding a strong stratification with the density contrast between top and bottom CZs about 700 .

Figure 1a shows the initial profiles of the density (solid) and temperature (dashed) of our model. The density profile in the range $0.71 \leq r / R_{\odot} \leq 0.991$ of the standard solar model is also shown in such a way as to fit the computational domain (dash-dotted) (Model S: Christensen-Dalsgaard et al. 1996). Our model has a stratification almost encompassing the solar CZ except its uppermost part.

All the variables are assumed to be horizontally periodic. Stress-free boundary conditions are used in the vertical direction for the velocity. Perfect conductor and vertical field conditions are used for the magnetic field at the bottom and top boundaries. A constant energy flux which drives the thermal convection is imposed on the bottom boundary, while the specific internal energy is fixed at the top boundary.

The fundamental equations are solved by the second-order Godunov-type finite-difference scheme that employs an approximate MHD Riemann solver (Sano et al. 1998). The magnetic field evolves with the Consistent MoC-CT method (Evans \& Hawley 1988, Clarke 1996). Non-dimensional parameters $\mathrm{Pr}=20, \mathrm{Pm}=2, \mathrm{Ra}=6 \times 10^{7}$, angular velocity of $\Omega_{0}=0.5$ and the spatial resolution of $\left(N_{x}, N_{y}, N_{z}\right)$ $=(256,256,256)$ are adopted, where the Prandtl, magnetic Prandtl, and Rayleigh numbers are defined by

$$
\operatorname{Pr}=\frac{\nu_{0}}{\left(\kappa_{0} / \rho c_{p}\right)}, \operatorname{Pm}=\frac{\nu_{0}}{\eta_{0}}, \operatorname{Ra}=\frac{g_{0} d_{\mathrm{cz}}^{4}}{\chi_{0} \nu_{0}} \frac{\delta}{H_{p}},
$$

where $\rho, \delta$, and $H_{p}$ are evaluated at $z=d_{\mathrm{cz}} / 2$.

In the following, the volume-, horizontal-, $x$ - and $y$-averages are denoted by single angular brackets with subscripts "v", "h", "x" and "y", respectively. The time-average of each spatial mean is denoted by additional angular brackets. The relative importance of convection to the rotation is measured by the Rossby number Ro $=\left(u_{\mathrm{cv}} k_{f}\right) /\left(2 \Omega_{0}\right)$, where $u_{\mathrm{cv}} \equiv$ $\sqrt{\left\langle\left\langle u_{z}^{2}\right\rangle_{\mathrm{v}}\right\rangle}$ is the mean convective velocity, and $k_{f}=2 \pi / d_{\mathrm{cz}}$. The global convective turn-over time and the equipartition field strength are defined by $\tau_{\mathrm{cv}} \equiv 1 /\left(u_{\mathrm{cv}} k_{f}\right)$ and $B_{\text {eq }}(z) \equiv$ $\sqrt{\left\langle\mu_{0} \rho u^{2}\right\rangle_{\mathrm{h}}}$. Note that $B_{\text {eq }}(z)$ is evaluated from the local convective energy and thus has a depth-dependence.

Since the sound speed in the deep CZ becomes very large in
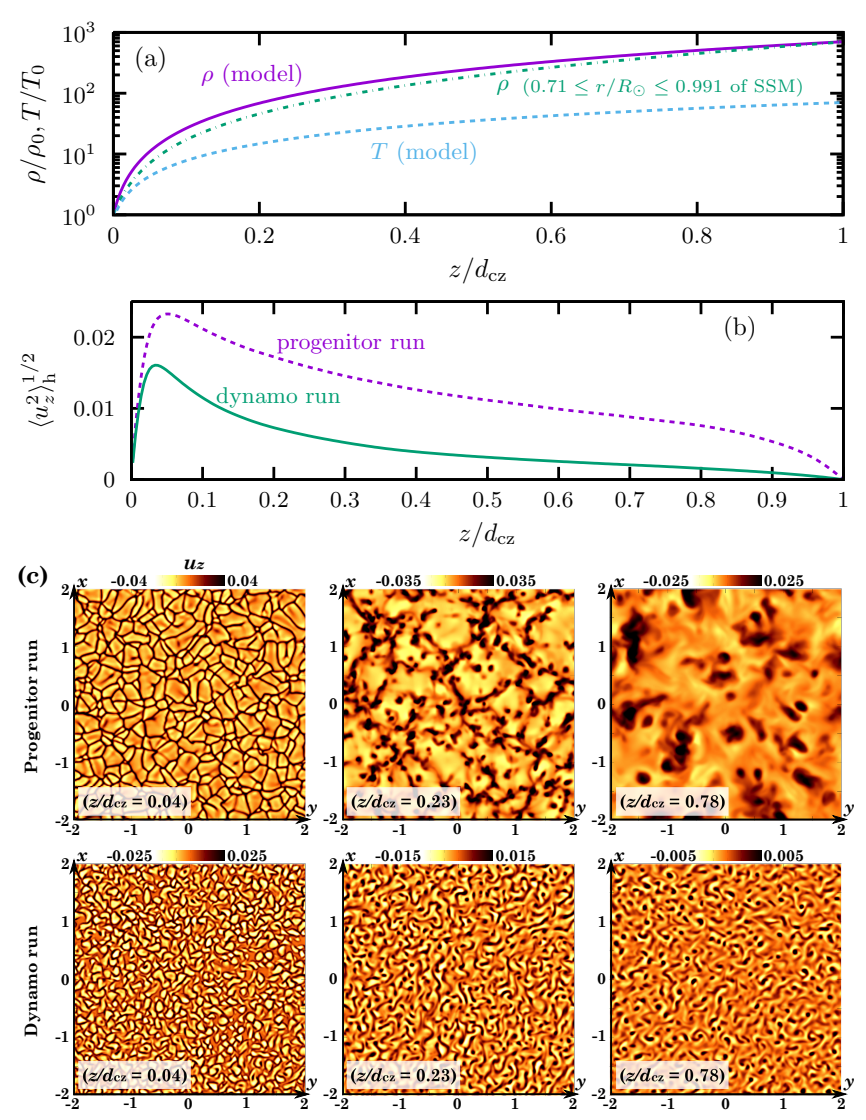

FIG. 1.- (a) Vertical profiles of the initial density (solid) and temperature (dashed) of the simulation model, and the density profile of the $\mathrm{CZ}$ of the standard solar model (dash-dotted). Normalization units are their surface values. (b) Vertical profiles of $\left\langle\left\langle u_{z}^{2}\right\rangle\right\rangle_{\mathrm{h}}^{1 / 2}$ for the progenitor (dashed) and dynamo (solid) runs. (c) The horizontal distributions of the $u_{z}$ at $z / d_{\mathrm{cz}}=0.04,0.23,0.78$ of the progenitor (upper) and dynamo (lower) runs.

the strongly-stratified model and imposes a strict limit on the time-step, a long thermal relaxation time is required in our fully-compressible simulation. To alleviate it, we first construct a progenitor model, in which the convection reaches a fully-developed state and the system becomes thermallyrelaxed, by evolving a non-rotating hydrodynamic run for $800 \tau_{\mathrm{cv}}$.

Shown in Figure $1 \mathrm{~b}$ and $1 \mathrm{c}$ are the vertical profile of $\left\langle\left\langle u_{z}^{2}\right\rangle\right\rangle_{\mathrm{h}}^{1 / 2}$ (dashed) and the distributions of $u_{z}$ in the $x-y$ plane at $z / d_{\mathrm{cz}}=0.04,0.23,0.78$ in the equilibrated state of the progenitor model. The black (orange) tone denotes down(up-)flows. The multi-scale convection with the strong updown asymmetry, i.e., the slower and broader upflow cell surrounded by networks of the faster and narrower downflow lanes, is developed in the progenitor model (e.g., Spruit et al. 1990; Miesch 2005). The dynamo run is started by adding the rotation and a seed weak horizontal field to the progenitor model.

\section{SIMULATION RESULTS}

\subsection{Basic Properties of Convection and Dynamo}

The temporal evolutions of $\epsilon_{\mathrm{K}} \equiv\left\langle\rho u^{2} / 2\right\rangle_{\mathrm{v}}, \epsilon_{\mathrm{Mt}} \equiv$ $\left\langle B^{2} / 2 \mu_{0}\right\rangle_{\mathrm{v}}$ and $\epsilon_{\mathrm{Mm}} \equiv\left(\left\langle B_{x}\right\rangle_{\mathrm{v}}^{2}+\left\langle B_{y}\right\rangle_{\mathrm{v}}^{2}\right) / 2 \mu_{0}$ (the energy of mean magnetic components) are shown by solid, dashed and dash-dotted lines in Figure 2a. The evolution of the $\epsilon_{\mathrm{K}}$ of the progenitor run before starting the dynamo run is also shown. 

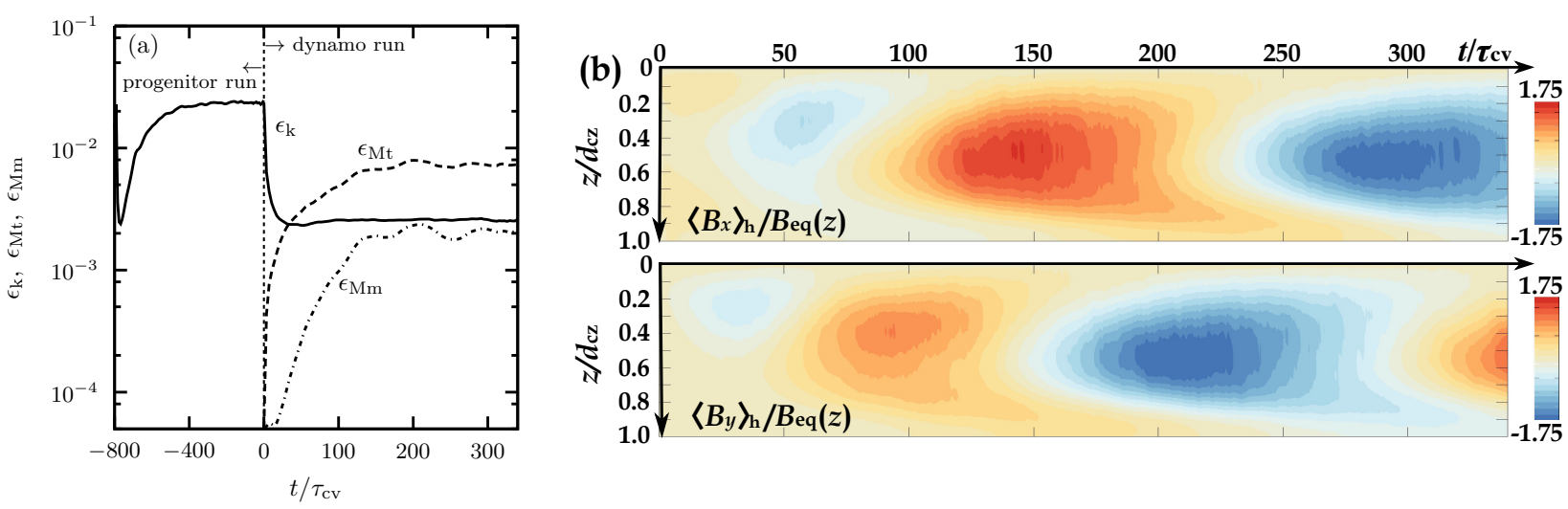

FIG. 2.- (a) The temporal evolution of $\epsilon_{\mathrm{K}}$ (solid), $\epsilon_{\mathrm{Mt}}$ (dashed) and $\epsilon_{\mathrm{Mm}}$ (dash-dotted). (b) Time-depth diagrams of $\left\langle B_{x}\right\rangle_{\mathrm{h}} / B_{\mathrm{eq}}(z)$ and $\left\langle B_{y}\right\rangle_{\mathrm{h}} / B_{\mathrm{eq}}(z)$.

Note that, from the horizontal symmetry and $\operatorname{div} B=0,\left\langle B_{z}\right\rangle_{\mathrm{h}}$ and $\left\langle B_{z}\right\rangle_{\mathrm{v}}$ are zero independent of time.

After a short relaxation time, the convective kinetic energy reaches a quasi-steady state at $t \simeq 50 \tau_{\mathrm{cv}}$. The magnetic energy is gradually amplified by the convection and is saturated at $t \simeq 120 \tau_{\mathrm{cv}}$. The mean values evaluated there are $u_{\mathrm{cv}}=5.9 \times$ $10^{-3}$ and $B_{\mathrm{eq}, \mathrm{v}} \equiv \sqrt{\left\langle\left\langle\mu_{0} \rho u^{2}\right\rangle\right\rangle_{\mathrm{v}}}=7.2 \times 10^{-2}$, providing $\tau_{\mathrm{cv}}=$ 54 and Ro $=0.02$. The vertical profile of $\left\langle\left\langle u_{z}^{2}\right\rangle\right\rangle_{\mathrm{h}}^{1 / 2}$ (solid) and the distributions of the $u_{z}$ on the horizontal planes in the dynamo-saturated stage are also shown in Figure $1 \mathrm{~b}$ and 1c. Since the rotation gives rise to the Coriolis force acting on the convective motion, the convective cell shrinks and thus the scale separation becomes larger in the rotating system. Since the mean kinetic helicity, which is a prerequisite for exciting the large-scale dynamo, arises as a natural consequence of the rotation, the dynamo-generated magnetic field also affects the convective motion shown in Figure 1c.

Figure $2 \mathrm{~b}$ shows the time-depth diagrams of $\left\langle B_{x}\right\rangle_{\mathrm{h}}$ and $\left\langle B_{y}\right\rangle_{\mathrm{h}}$ normalized by $B_{\text {eq }}(z)$. Note that the turbulent magnetic component is eliminated by taking horizontal average. It is found that the oscillatory large-scale horizontal magnetic component is spontaneously organized in the bulk of the CZ. It has a peak with the super-equipartition strength in the mid-part of the $\mathrm{CZ}$ and propagates from there to the top and base of the CZ. Since there exists a phase difference of $\pi / 2$ between $\left\langle B_{x}\right\rangle_{\mathrm{h}}$ and $\left\langle B_{y}\right\rangle_{\mathrm{h}}$, the mean horizontal magnetic flux, defined by $B_{h} \equiv \sqrt{\left\langle B_{x}\right\rangle_{\mathrm{h}}^{2}+\left\langle B_{y}\right\rangle_{\mathrm{h}}^{2}}$, has a quasi-steady vertical profile.

The large-scale dynamo observed here in the stronglystratified model has physical properties similar to those in the weakly-stratified convective dynamo simulations (e.g., Käpylä et al. 2013, MS14a). Because of the horizontal symmetry and thus no differential rotation in our system, the turbulent electro-motive force would be solely responsible for the dynamo (see MS14b for a mean-field $\alpha^{2}$-dynamo model which can quantitatively reproduce the DNS results). Our intriguing finding in this Letter, which have not been observed in the weakly-stratified model with the similar boundary conditions, is spontaneous formation of large-scale magnetic structures in the $\mathrm{CZ}$ surface, which will be reported in the following.

\subsection{Spontaneous Formation of Surface Magnetic Structure}

A series of snapshots where the distribution of the $B_{z}$ at different time on the horizontal cutting plane at $z / d_{\mathrm{cz}}=0.04$ is shown in the top panel of Figure 3a. The darker (lighter) tone denotes positive (negative) $B_{z}$. While the $B_{z}$ has a small- scale tangled structure with the typical size comparable to the convective cell in the initial evolutionary stage [(a1)(a2)], it evolves as time passes to organize the large-scale structure with the spatial-scale much larger than the convective cell [(a3)-(a4)]. The surface magnetic structure has the dynamically-important strength comparable to $B_{\text {eq }}(z)$ and is recurrently-appeared in the dynamo-saturated stage [(a5)], implying that it should be a consequence of the stronglystratified MHD convection rather than a transiently-formed structure.

The one-sided horizontal average ( $x$ - or $y$-average) of the $B_{z}$ may be helpful to clearly demonstrate its depth-dependence because the horizontal average of it, i.e., $\left\langle B_{z}\right\rangle_{\mathrm{h}}$, is zero in our setup. Shown in lower panel of Figure $3 \mathrm{a}$ is a corresponding time series of snapshots for the distributions of the $\left\langle B_{z}\right\rangle_{\mathrm{y}}[(\mathrm{a} 1)-(\mathrm{a} 4)]$ or $\left\langle B_{z}\right\rangle_{\mathrm{x}}[(\mathrm{a} 5)]$ in units of $B_{\text {eq }}(z)$. The blue (red) tone represents the positive (negative) value. The initial small-scale vertical magnetic structure gradually evolves to the larger scale in the upper $\mathrm{CZ}$. While it prevails in the mid-part of the $\mathrm{CZ}$ in the dynamo-saturated stage, the core of the large-scale $B_{z}$ structure stays in around the $\mathrm{CZ}$ surface.

Figure $3 \mathrm{~b}$ shows the temporal evolution of two-dimensional (2D) Fourier spectrum of the $B_{z}^{2}$ in the upper CZ. Here the spectrum at each depth is projected onto a one-dimensional wavenumber $k^{2}=k_{x}^{2}+k_{y}^{2}$ and then is averaged over the normalized depth from 0.0 to 0.25 . The energy-containing scale of the $B_{z}$ becomes larger with the time and finally reaches the possible largest scale in the upper $\mathrm{CZ}$, confirming the existence of the large-scale $B_{z}$ structure in the upper CZ. Since the large-scale vertical magnetic component cannot be generated solely by the $\alpha^{2}$-type dynamo only with the vertical helicity variation (see, MS14b), some additional mechanism should play a role in organizing it in this simulation.

\subsection{What Makes Surface Magnetic Structure Organized?}

The so-called negative magnetic pressure instability (NEMPI) has been proposed as a mechanism for the selfassembly of the magnetic flux near the CZ surface (e.g., Kleeorin et al. 1996, Brandenburg et al. 2010). Although its presence has been confirmed numerically in the forced MHD turbulence (Brandenburg et al. 2011, 2013, Mitra et al. 2014, Jabbari et al. 2014; Warnecke et al. 2013), it would not play a significant role in organizing the surface magnetic structure in our simulation. This is because the relatively-rapid rotation with $\mathrm{Ro}=0.02$ is assumed here, but according to Losada et al. (2012), Ro $\gtrsim 5$ is required for exciting the NEMPI. Here possible two alternatives are discussed as a cause of the mag- 

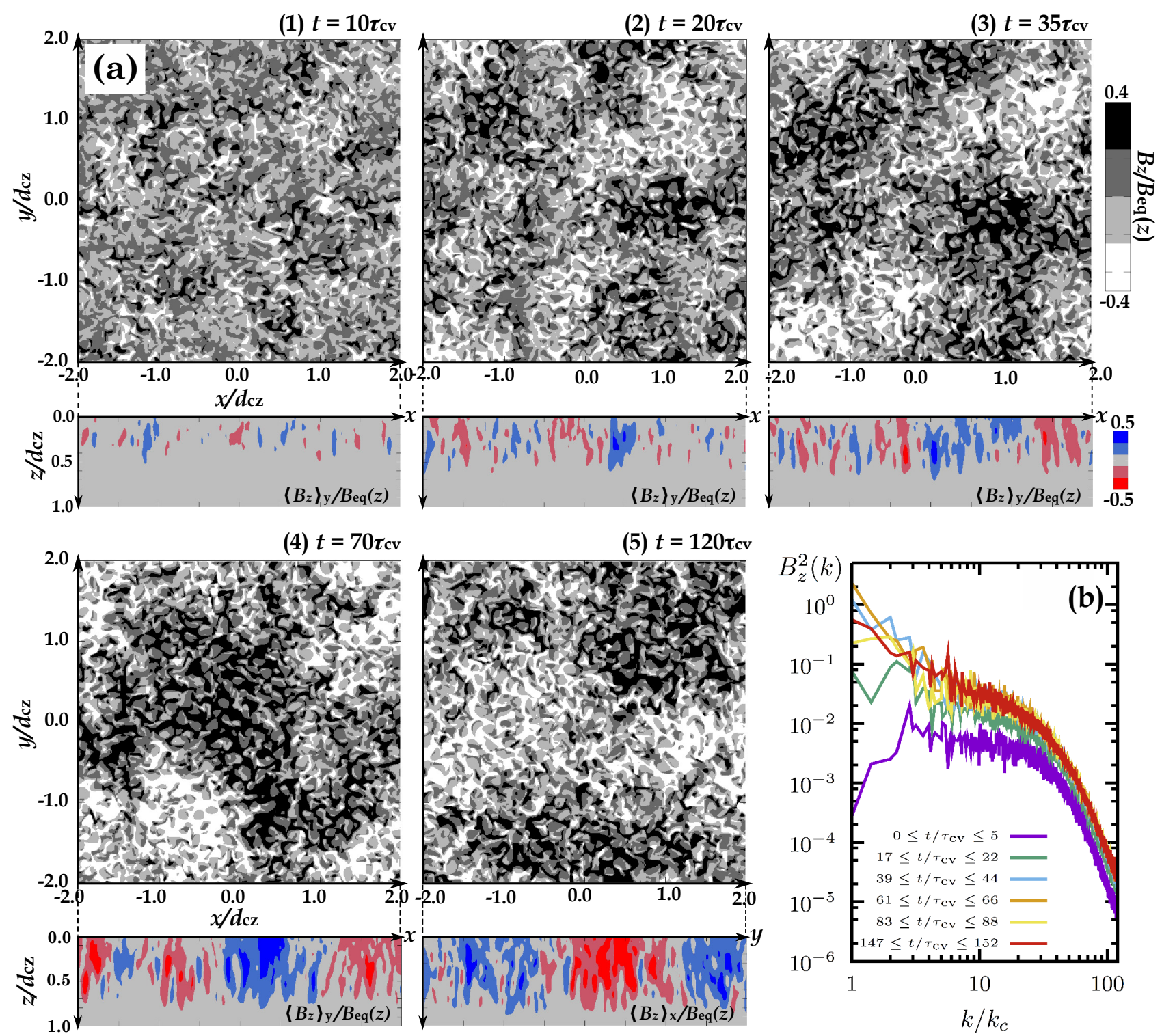

FIG. 3.- (a) A series of snapshots for the horizontal distribution of the $B_{z}$ at $z / d_{\mathrm{cz}}=0.04$ and vertical distribution of the $\left\langle B_{z}\right\rangle_{\mathrm{y}} / B_{\mathrm{eq}}(z)$ or $\left\langle B_{z}\right\rangle_{x} / B_{\mathrm{eq}}(z)$. (b) The temporal evolution of $2 \mathrm{D}$ Fourier spectrum of the $B_{z}^{2}$ in the upper $\mathrm{CZ}$, where $k_{c}=2 \pi / W$.

netic structure formation: one is the Parker instability (Parker 1979) and the other is the flux expulsion accompanied with the strong downflow (e.g., Weiss 1966; Kitiashvili et al.2010, Stein \& Nordlund 2012; Käpylä et al. 2015).

Figure 4a shows the growth rate of the Parker instability obtained from the WKB dispersion equation

$$
\begin{aligned}
\left(c_{\star}^{2}+v_{A}^{2}\right) \gamma^{4} & +v_{A}^{2}\left[2\left(c_{\star}^{2}+v_{A}^{2}\right) k_{h}^{2}+g_{0} \frac{D}{D z} \ln \left(\frac{B_{h}}{\rho}\right)\right] \gamma^{2} \\
+ & k_{h}^{2} v_{A}^{4}\left(k_{h}^{2} c_{\star}^{2}+g_{0} \frac{D}{D z} \ln B_{h}\right)=0,
\end{aligned}
$$

(e.g., Gilman 1970), where $\gamma$ is the growth rate, $k_{h}$ is the horizontal wavenumber, $B_{h}$ is the horizontal magnetic flux, $c_{\star} \equiv \sqrt{P / \rho}$, and $v_{A} \equiv B_{h} / \sqrt{\mu_{0} \rho}$. For deriving the depthdependent growth rate, we adopt $P=\langle P\rangle_{\mathrm{h}}, \rho=\langle\rho\rangle_{\mathrm{h}}$ and $B_{h}=\sqrt{\left\langle B_{x}\right\rangle_{\mathrm{h}}^{2}+\left\langle B_{y}\right\rangle_{\mathrm{h}}^{2}}$ evaluated from the simulation model. The different line-type denotes the growth rate at the differ- ent depth. The vertical and horizontal axes are normalized by $1 / \tau_{\mathrm{cv}}$ and $k_{c}=2 \pi / W$. Note that the instability is inhibited in the range $k / k_{c}<1$ due to the box-width constraint.

The dynamo-maintained magnetic flux is unstable to the Parker instability in the span $0 \leq z / d_{\mathrm{cz}} \lesssim 0.4$. Since the typical growth time of it is comparable to or a bit smaller than $\tau_{\mathrm{cv}}$, it has a sufficient time to grow during the simulation. However, the most unstable mode has a smaller wavelength in comparison with the box-width, implying the difficulty to explain the dominance of the box-sized surface magnetic structure in our simulation.

Additionally to the mismatch of the magnetic spatial-scales, the growth of the Parker instability itself may be inhibited by vigorous convective motions. In Figure $4 b$, the growth time of the most unstable mode of the Parker instability (solid: $\left.\tau_{\mathrm{pk}, \max }\right)$ and the local convective turn-over time defined by $\tau_{\mathrm{cv}, \mathrm{l}}(z)=H_{\rho}(z) /\left\langle u_{z}^{2}\right\rangle_{\mathrm{h}}^{1 / 2}$ (dashed) are compared as a function of the depth, where $H_{\rho} \equiv \mathrm{d} z / \mathrm{d} \ln \langle\rho\rangle_{\mathrm{h}}$. In the upper $\mathrm{CZ}$, the 

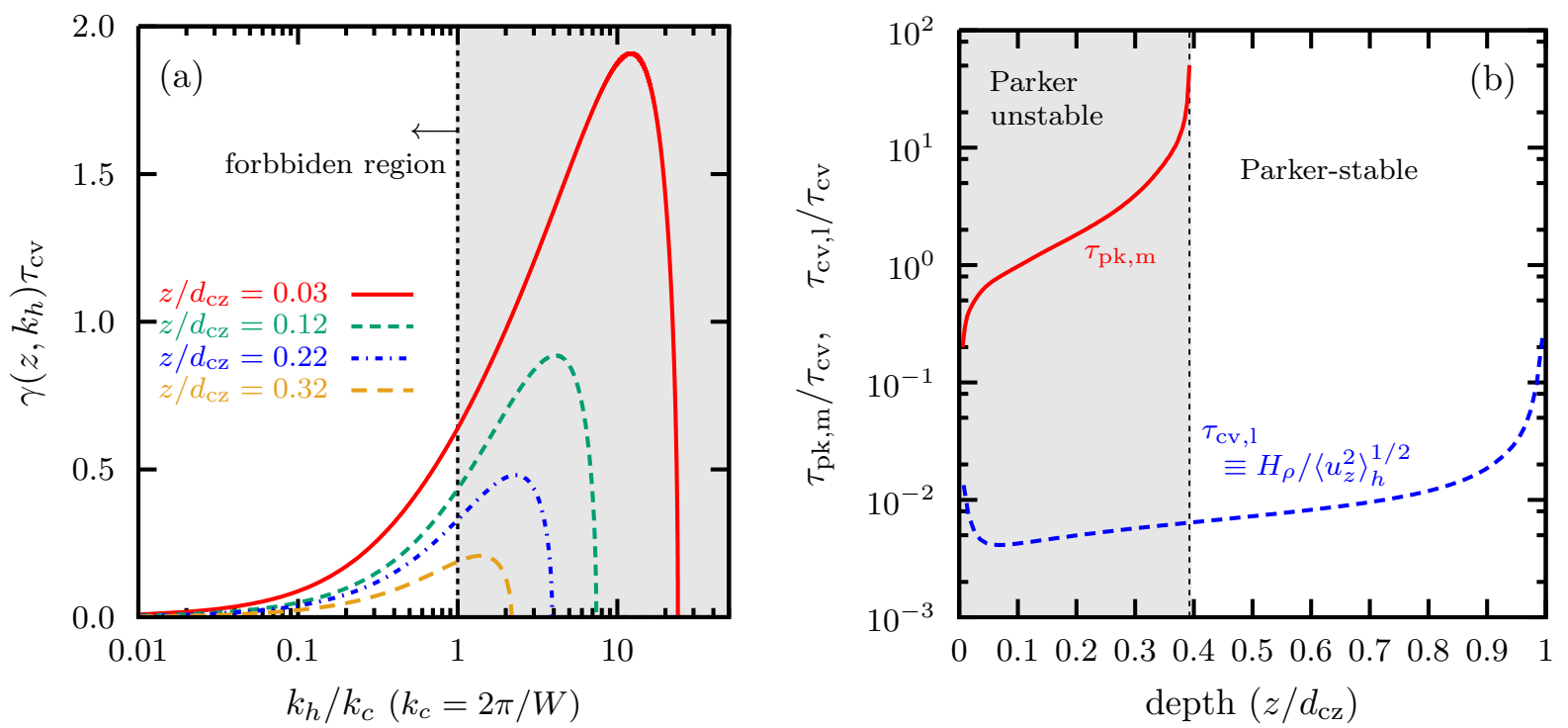

FIG. 4.- (a) The growth rate of the Parker instability evaluated from the simulation result. (b) The comparison between the growth time of the fastest-growing mode of the Parker instability $\left(\tau_{\mathrm{pk}, \mathrm{m}}\right)$ and the local convective turn-over time $\left(\tau_{\mathrm{cv}, \mathrm{l}}\right)$ as a function of the depth.

condition $\tau_{\mathrm{cv}, 1} \ll \tau_{\mathrm{pk} \text {,max }}$ is always satisfied. Since, in such a situation, the small-scale convective motion violently disturbs the coherency of the magnetic flux, we would have to say that the Parker instability would not be responsible for the largescale structure formation observed in our simulation.

Next, the large-scale flow and its association with the surface magnetic structure are analyzed. For casting light on the large-scale pattern, the small-scale structures with $k / k_{c} \gtrsim 8$ are eliminated by applying Fourier filtering (e.g., Warnecke et al. 2015, Jabbari et al. 2016). A series of snapshots where $\left\langle B_{z}\right\rangle_{k_{8}}$ and $\left\langle u_{z}\right\rangle_{k_{8}}$ on the horizontal plane at $z / d_{\mathrm{cz}}=0.04$ are shown in Figure $5 \mathrm{a}$, where $\langle\cdot\rangle_{k_{8}}$ denotes Fourier filtering. The over-plotted arrows are the velocity vectors composed of $\left\langle u_{x}\right\rangle_{k_{8}}$ and $\left\langle u_{y}\right\rangle_{k_{8}}$. Additionally, 2D spectra of $B_{h}^{2}, B_{z}^{2}, \rho v_{h}^{2}$ and $\rho v_{z}^{2}$ in the upper CZ are also shown in Figure 5b. The spectrum at each depth is spatially-averaged over the normalized depth from 0.0 to 0.25 and is temporally-averaged over $10 \tau_{\mathrm{cv}}$ around the corresponding reference time.

It is prominent that, in the dynamo-saturated stage, the bipolar "band-like" structure elongated along the direction of the horizontal magnetic flux is predominant (see Fig.2b). Although the faster horizontal flow and stronger downflow can be found in/around the region with the stronger $B_{z}$ before the dynamo-saturation (left column), large-scale flow pattern is not necessarily associated with the magnetic structure in the dynamo-saturated stage (middle and right columns). In addition, we can find from the spectra that the energy contained in the large-scale magnetic components is much larger than that of the large-scale flow in the upper CZ. This would suggest that the large-scale flows are not the cause, but a consequence of the large-scale magnetic structures in the upper CZ.

Overall our analyses indicate that there should be an as-yetunknown mechanism for the self-organization of large-scale magnetic structures, which would be inherent in the stronglystratified atmosphere. The band-like magnetic structure observed in our simulation is similar to that observed in the large-scale dynamo by the forced turbulence in a stronglystratified atmosphere (Mitra et al. 2014; Jabbari et al. 2016). This may imply that the surface magnetic structure formation is a common universal feature of the strongly-stratified MHD turbulence regardless of its details.

\section{SUMMARY}

In this Letter, we studied numerically MHD convections in the strongly-stratified atmosphere resembling the solar interior. The large-scale dynamo observed in our simulation had physical properties similar to those observed in the weaklystratified model (see MS14ab): the oscillatory large-scale horizontal magnetic component with the dynamically-important strength was spontaneously organized in the bulk of the CZ. Its spatio-temporal evolution strongly suggests that the $\alpha^{2}$ type mechanism would be responsible for the MHD dynamo in our system.

Our intriguing finding, which have not been observed in the weakly-stratified model, was the spontaneous formation of the large-scale $B_{z}$ structure in the CZ surface. Small-scale tangled components of the $B_{z}$ which was seen in the earlier evolutionary stage gradually evolved to the large-scale organized structure with the size much larger than convective cell as time passes in the upper CZ. In the dynamo-saturated stage, the bipolar "band-like" $B_{z}$ structure was predominant and was recurrently-appeared. Since the possible candidates, such as the NEMPI, Parker instability, and flux expulsion due to the strong downflow, had difficulties to explain the surface magnetic structure formation, our results may suggest the existence of an as-yet-unrecognized mechanism inducing the spontaneous formation of the large-scale magnetic structure in the strongly-stratified convective atmosphere.

The large-scale dynamo observed here maintains the quasisteady magnetic flux and thus is different from the actual solar dynamo with a quasi-periodic flux modulation. Furthermore, since we adopt the faster rotation than that achieved in the actual Sun and ignore the global effects, such as differential rotation and meridional flows, our model still remains a long way from the solar interior. However, despite a lack of some solar elements, it would be interesting that the relatively shallow root of the surface magnetic structure, implied from our simulation, seems to be compatible with some observations of the magnetic patches on the solar surface (e.g., Brandenburg 2005, Hara 2009). Understanding the self-organization mech- 

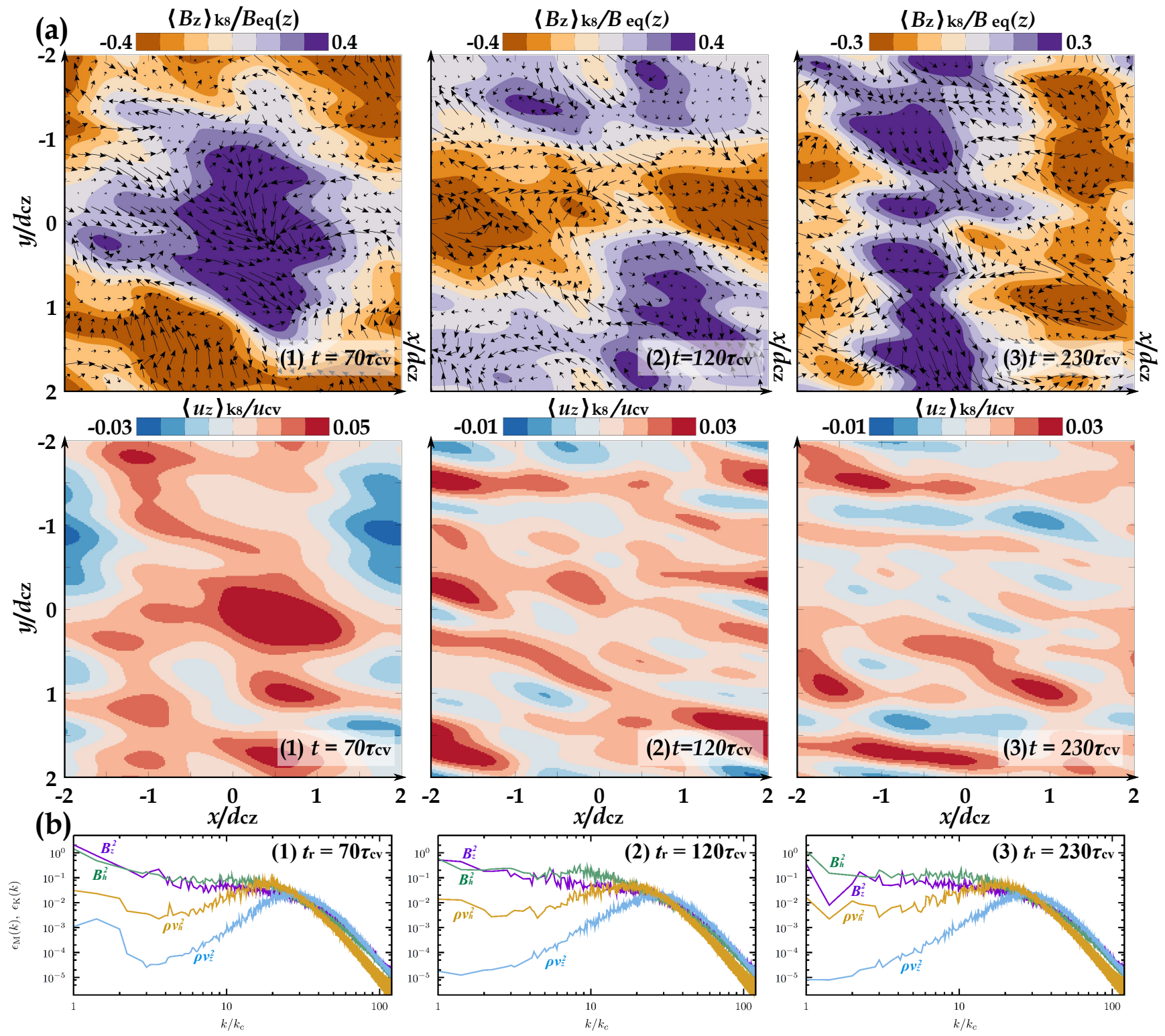

FIG. 5.- (a) A series of snapshots of $\left\langle B_{z}\right\rangle_{k_{8}}$ and $\left\langle u_{z}\right\rangle_{k_{8}}$ on the horizontal plane at $z / d_{\mathrm{cz}}=0.04$. The over-plotted arrows are the velocity vectors. (b) $2 \mathrm{D}$ spectra of $B_{h}^{2}, B_{z}^{2}, \rho v_{h}^{2}$ and $\rho v_{z}^{2}$ in the upper $\mathrm{CZ}$ at around the reference time $t_{r}$.

anism of the surface magnetic structure observed in our study should deepen our knowledge of the solar magnetism and is a priority target of our future work.

We acknowledge an anonymous referee for constructive comments. Computations were carried out on XC30 at NAOJ. This work was supported by JSPS KAKENHI Grant number $15 \mathrm{~K} 17611$ and the joint research project of the Institute of Laser Engineering, Osaka University.

\section{REFERENCES}

Augustson, K., Brun, A.S., Miesch, M., \& Toomre, J. 2015, ApJ, 809, 149 Brandenburg, A. 2005, ApJ, 625, 539

Brandenburg, A., Kleeorin, N., \& Rogachevskii, I. 2010, Astronomische Nachrichten, 331, 5

Brandenburg, A., Kemel, K., Kleeorin, N., Mitra, D., \& Rogachevskii, I. 2011, ApJ, 740, L50

Brandenburg, A., Kleeorin, N., \& Rogachevskii, I. 2013, ApJ, 776, L23

Charbonneau, P. 2010, Living Reviews in Solar Physics, 7, 3

Cheung, M.C.M., Rempel, M., Title, A.M., \& Schüssler, M. 2010, ApJ, 720, 233

Christensen-Dalsgaard, J., et al. 1996, Science, 272, 1286

Clarke, D.A. 1996, ApJ, 457, 291
Evans, C.R., \& Hawley, J.F. 1988, ApJ, 332, 659

Fan, Y., \& Fang, F. 2014, ApJ, 789, 35

Ghizaru, M., Charbonneau, P., \& Smolarkiewicz, P.K. 2010, ApJ, 715, L133 Gilman, P. A. 1970, ApJ, 162, 1019

Hara, H. 2009, ApJ, 697, 980

Jabbari, S., Brandenburg, A., Losada, I. R., Kleeorin, N., \& Rogachevskii, I. 2014, A\&A, 568, A112

Jabbari, S., Brandenburg, A., Mitra, D., Kleeorin, N., \& Rogachevskii, I. 2016, arXiv:1601.08167

Käpylä, P.J., Mantere, M.J., \& Brandenburg, A. 2012, ApJ, 755, L22

Käpylä, P.J., Mantere, M.J., \& Brandenburg, A. 2013, GAFD, 107, 244 
Käpylä, P.J., Brandenburg, A., Kleeorin, N., Käpylä, M.J., \& Rogachevskii, I. 2015, arXiv: 1511.03718

Kitiashvili, I.N., Kosovichev, A.G., Wray, A.A., \& Mansour, N.N. 2010, ApJ, 719, 307

Kleeorin, N., Mond, M., \& Rogachevskii, I. 1996, A\&A, 307, 293

Losada, I.R., Brandenburg, A., Kleeorin, N., Mitra, D., \& Rogachevskii, I. 2012, A\&A, 548, A49

Masada, Y., Yamada, K., \& Kageyama, A. 2013, ApJ, 778, 11

Masada, Y., \& Sano, T. 2014 PASJ, 66, S2

Masada, Y., \& Sano, T. 2014, ApJ, 794, L6

Miesch, M.S. 2005, Living Reviews in Solar Physics, 2,1

Mitra, D., Brandenburg, A., Kleeorin, N., \& Rogachevskii, I. 2014, MNRAS, 445, 761
Nelson, N.J., Brown, B.P., Brun, A.S., Miesch, M.S., \& Toomre, J. 2013, ApJ, 762, 73

Parker, E. N. 1979, Oxford University Press, 1979, 858 p.,

Rempel, M., \& Cheung, M.C.M. 2014, ApJ, 785, 90

Sano, T., Inutsuka, S., \& Miyama, S. M. 1998, ApJ, 506, L57

Spruit, H.C., Nordlund, A., \& Title, A.M. 1990, ARA\&A, 28, 263

Stein, R.F., \& Nordlund, Å. 2012, ApJ, 753, L13

Warnecke, J., Losada, I. R., Brandenburg, A., Kleeorin, N., \& Rogachevskii, I. 2013, ApJ, 777, L37

Warnecke, J., Losada, I. R., Brandenburg, A., Kleeorin, N., \& Rogachevskii, I. 2015, arXiv: 1502.03799

Weiss, N.O. 1966, Proceedings of the Royal Society of London Series A, 293, 310

Yadav, R.K., Gastine, T., Christensen, U.R., \& Reiners, A. 2015, A\&A, 573, A68 